\title{
Phenomenological description of scaling laws of sediment transport
}

\author{
Subhasish Dey* and Sk Zeeshan Ali \\ Department of Civil Engineering, Indian Institute of Technology Kharagpur, Kharagpur 721302, \\ India
}

\begin{abstract}
In this paper, we seek the scaling laws of sediment transport under a turbulent flow by applying the phenomenological theory of turbulence. The results show that at the threshold of sediment motion, the densimetric Froude number follows a " $(1+\sigma) / 4$ " scaling law with the relative roughness number (ratio of particle size to flow depth), where $\sigma$ is the spectral exponent. For the bedload transport, the bedload transport intensity follows a " $3 / 2$ " and " $(1+\sigma) / 4$ " scaling laws with the transport stage function and the relative roughness, respectively. For the scour in a contracted stream, the dimensionless scour depth follows a " $4 /(3-\sigma)$ ", "$4 /(3-\sigma)$ " and " $-(1+\sigma) /(3-\sigma)$ " scaling laws with the densimetric Froude number, the channel contraction ratio and the relative roughness, respectively.
\end{abstract}

\section{Introduction}

The phenomenological theory of turbulence was accredited to Kolmogorov's [1] pioneering contributions to the scaling laws of the fully developed homogeneous and isotropic turbulence. The phenomenological theory allows us to anticipate the scaling laws of classical problems of sediment transport in a simplified way demanding less heuristic arguments. The advantage of this theory to analyse a problem is that this theory provides a universal relationship, unlike the empirical laws, by linking the dependent and the independent variables. However, this theory cannot predict the multiplicative constant linking the dependent and the independent variables and thus, the multiplicative constant must be obtained from experimental data. The applications of the phenomenological theory of turbulence in modelling the turbulent flows received much attention in the past [2]. Some of them include (i) counting degrees of freedom, (ii) linking the microscopic and the macroscopic scales and (iii) deriving the probability distribution function of velocity gradients and many others. The phenomenological theory of turbulence was also applied to obtain the similarity laws in open-channel flows [3]. Here, we make an attempt to explore the scaling laws of sediment transport from the perspective of the phenomenological theory of turbulence. We apply the laws of turbulent energy spectrum together with the momentum transfer theory to find the link between the laws of sediment transport and the turbulent energy spectrum.

\footnotetext{
* Corresponding author: sdey@iitkgp.ac.in
} 
The paper is arranged as follows. First, a brief description of the spectral laws of turbulence is presented. Then, the scaling laws of the threshold velocity, the bedload transport rate and the scour in a contracted stream are derived. Finally, concluding remarks are made.

\section{Spectral laws of turbulence}

The velocity scale $v_{l}$ of a turbulent eddy with a length scale $l$ is expressed as

$$
v_{l} \sim\left(\int_{1 / l}^{\infty} E(k) \mathrm{d} k\right)^{1 / 2}
$$

where $E(k)$ is the energy spectrum function, $k$ is the eddy wavenumber and the symbol " $\sim$ " signifies "scales with". The $E(k)$ can be expressed as $E(k) \sim V_{L}^{2} L^{1+\sigma} k^{\sigma}$, where $V_{L}$ is the velocity scale of the large-scale eddies having a length scale $L$ and $\sigma$ is the spectral exponent. Substituting this form of $E(k)$ into Eq. (1) produces

$$
v_{l} \sim V_{L}\left(\frac{l}{L}\right)^{-(1+\sigma) / 2}
$$

When the large scale and the small scale are separated in the $E(k)$ plane, it creates two inertial ranges, namely the energy inertial range and the enstrophy inertial range [4]. In a two-dimensional (2D) turbulence, two plausible cascades exist: (i) the energy inverse cascade and (ii) the enstrophy cascade. In the energy inverse cascade, energy flows from small to large scales; while in the enstrophy cascade, the enstrophy flows from large to small scales. The 2D turbulence, unlike the three-dimensional (3D) turbulence, is not featured by the vortex stretching. The energy inverse cascade produces: $E(k) \sim \varepsilon^{2 / 3} k^{-5 / 3}$, where $\varepsilon$ is the turbulent kinetic energy (TKE) dissipation rate. By contrast, the enstrophy cascade produces: $E(k) \sim \beta^{2 / 3} k^{-3}$, where $\beta$ is the enstrophy dissipation rate. Another spectral law for a wall-bounded turbulent flow states: $E\left(L^{-1} \leq k \leq z^{-1}\right) \sim u_{*}^{2} k^{-1}$ [5], where $u *$ is the shear velocity and $z$ is the vertical distance. Importantly, for both $2 \mathrm{D}$ and $3 \mathrm{D}$ turbulence, the " $-5 / 3$ " spectral law in the energy inertial range is legitimate irrespective of the direction of energy transfer.

In subsequent sections, we seek the scaling laws of three classical problems of sediment transport by applying the phenomenological theory of turbulence. They include the determination of the scaling laws of threshold velocity, bedload transport rate and scour in a contracted stream.

\section{Scaling law of threshold velocity}

In fluvial hydrodynamics, a threshold velocity is referred to the average flow velocity $U_{c}$ which is sufficient to begin the sediment particle motion at the bed surface. This phenomenon is called the threshold of sediment motion [6]. 


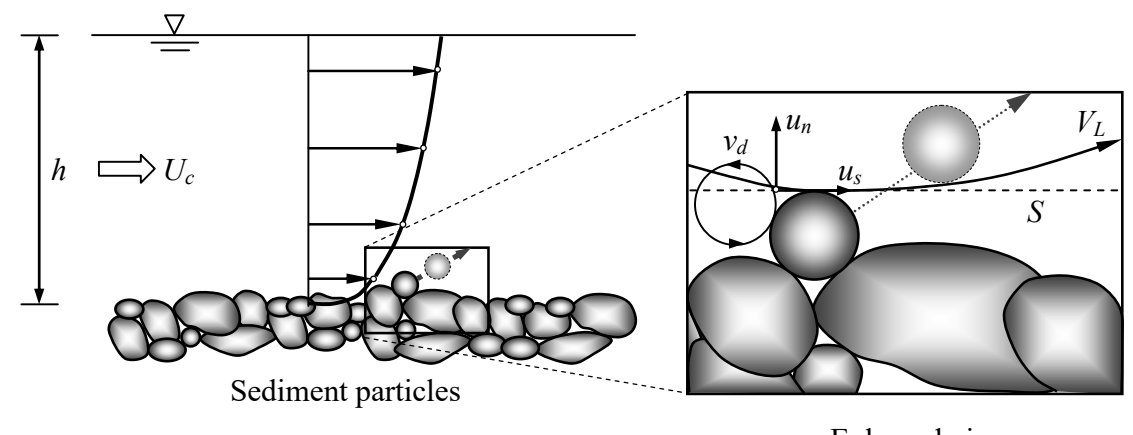

Enlarged view

Fig. 1. Sketch of threshold of sediment motion.

Figure 1 shows a conceptual representation of the threshold of sediment motion. Here, a turbulent stream, having a mean flow depth $h$ and mean flow velocity $U$, flows over a sediment bed that consists of sediment particles of size $d$. In previous empirical formulas given by several researchers, the nondimensional threshold velocity was expressed as $F_{d c}=$ $f(\zeta)$, where $F_{d c}\left[=U_{c} /(\Delta g d)^{1 / 2}\right]$ is the densimetric Froude number, $\Delta\left[=\left(\rho_{p}-\rho_{f}\right) / \rho_{f}\right]$ is the submerged relative density of sediment particles, $\rho_{p}$ is the mass density of sediment particles, $\rho_{f}$ is the mass density of fluid, $g$ is the gravitational acceleration, $\zeta(=d / h)$ is the relative roughness number and subscript ' $c$ ' denotes the threshold condition. Figure 1 provides an enlarged view of the bed particles depicting the fluid-sediment interaction. The Reynolds shear stress $\tau_{t}$ created due to the local eddy at a wetted surface $S$ tangential to the crest of the target particle is expressed as $\tau_{t}=\rho_{f} \overline{u_{s} u_{n}}$, where $u_{s}$ and $u_{n}$ are the velocity fluctuations tangential and normal to the $S$, respectively, and the over-line represents the time-averaging. The $\tau_{t}$ is in fact generated by the momentum transfer across the $S$. Above the $S$, the flow velocity scales with $V_{L}(\sim U)$. Therefore, the fluid conveys a considerable horizontal component of momentum per unit volume $\left(\sim \rho_{f} U\right)$ tangential to the $S$. The local eddy, bestriding the $S$, transfers the fluid of low and high momentum fluxes tangential to the $S$ in the upward and downward directions, respectively. The net momentum flux transfer across the $S$ is therefore accomplished by the velocity normal to the $S$. As a result, the $\tau_{t}$ becomes $\tau_{t} \sim \rho_{f} U u_{n}$ [3]. Since the eddies of sizes less than $l$ adjust within the inter particles space, they provide a significant contribution to the velocity normal to the $S\left(u_{n} \sim\right.$ $v_{d}$ as $l$ approached $d$ ). From Eq. (2), we obtain

$$
v_{d} \sim U\left(\frac{d}{h}\right)^{-(1+\sigma) / 2} \sim U \zeta^{-(1+\sigma) / 2}
$$

It produces

$$
\tau_{t} \sim \rho_{f} U v_{d} \sim \rho_{f} U^{2} \zeta^{-(1+\sigma) / 2}
$$

The $U_{c}$ can be obtained by equating the Reynolds shear stress developed at the $S$ and the threshold bed shear stress $\tau_{0 c}\left[=\Delta \rho_{f} g d \Theta_{c}\right]$, where $\Theta_{c}$ is the threshold Shields parameter. In fact, the $\Theta_{c}$, in functional form, can be expressed as $\Theta_{c}=G(D *)$, where $D *\left[=\left(d \Delta g / v^{2}\right)^{1 / 3}\right]$ is the particle parameter. At threshold of sediment motion, it follows:

$$
U_{c}^{2} \zeta^{-(1+\sigma) / 2} \sim \Delta g d G\left(D_{*}\right) \Rightarrow F_{d c} \sim \zeta^{(1+\sigma) / 4} G^{1 / 2}\left(D_{*}\right)
$$


Figure 2 depicts the $F_{d c}$ as a function of $\zeta$. The experimental data taken from several investigators [7-13] are also plotted. For $\sigma=-5 / 3$, Eq. (5) produces $F_{d c} \sim \zeta^{-1 / 6}$, which has a significant agreement with the experimental data over a wide range of $\zeta$. For $\zeta<10^{-4}$, the particle diameter approaches the viscous sublayer thickness and as a result, the momentum transfer becomes viscous. For this case, the enstrophy cascade $(\sigma=-3)$ becomes dominant resulting in $F_{d c} \sim \zeta^{-1 / 2}$. The experimental data, in general, corresponds to the "-1/2" scaling law for $10^{-6}<\zeta<10^{-4}$. On the other hand, when $\zeta$ becomes large $\left(\zeta \geq 10^{-1}\right)$, the turbulent energy spectrum obeys $\sigma=-1$ due to the presence of energy containing eddies. This results $F_{d c} \sim \zeta^{\curvearrowleft} G(D *)$, indicating that the $F_{d c}$ is independent of $\zeta$.

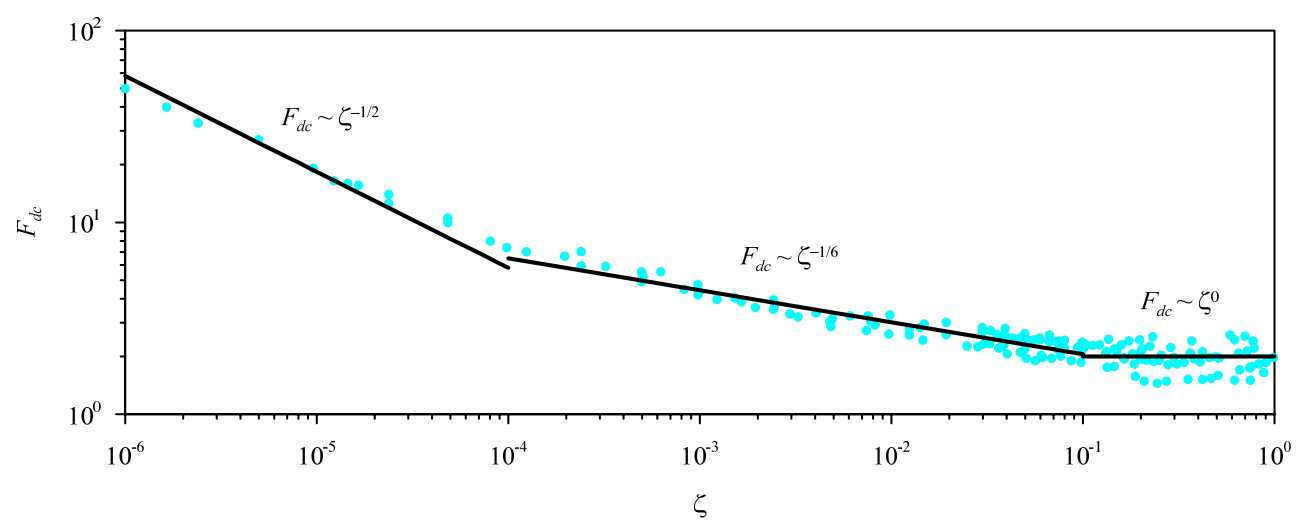

Fig. 2. The threshold densimetric Froude number $F_{d c}$ as a function of relative roughness $\zeta$.

\section{Scaling law of bedload transport rate}

When the flow velocity surpasses the threshold velocity, the sediment particles are set in motion. Under this circumstance, the applied bed shear stress $\tau_{0}$ exceeds the threshold bed shear stress $\tau_{0 c}$ and the transport of sediment particles occurs within a thin layer (known as the bedload layer), in the form of successive contacts of the particles with the bed. This phenomenon is called the bedload transport. The bedload transport rate $q_{b}$ can be scaled as $q_{b} \sim n d^{3} v_{p}$ [6], where $n$ is the number of particles per unit area and $v_{p}$ is the particle velocity. To seek a scaling law of $q_{b}$, we need to obtain the scaling laws for $n$ and $v_{p}$. According to the conventional mechanics of bedload transport [14], the applied bed shear stress $\tau_{0}$ can be decomposed into the dispersive particle shear stress $\tau_{0 p}$ and the interfacial fluid shear stress $\tau_{0 f}\left(\tau_{0}=\tau_{0 p}+\tau_{0 f}\right)$. Bagnold [14] considered that during the bedload transport, the $\tau_{0 f}$ equals the $\tau_{0 c}$. Thus, the component $\tau_{0 c}$ of the $\tau_{0}$ is directly transferred to the immobile bed particles in the form of skin frictional resistance. On the other hand, the residual bed shear stress $\left(\tau_{0}-\tau_{0 c}\right)$ is transferred to the mobile particles in the form of a drag induced bed shear stress $\tau_{0 b}$. Thus, $\tau_{0 p} \sim \tau_{0 b} \sim n f_{D}$ [15], where $f_{D}$ is the drag force. The $f_{D}$ can be scaled as $f_{D} \sim$ $\Delta \rho_{f} g d^{3}[6]$. Therefore,

$$
n \sim \frac{\tau_{0}-\tau_{0 c}}{f_{D}} \sim \frac{\tau_{0}-\tau_{0 c}}{\Delta \rho_{f} g d^{3}} \sim \frac{\Theta-\Theta_{c}}{d^{2}}
$$

Noting that the bed shear stress can be scaled with the square of the flow velocity, the relationship $\tau_{0 p}=\tau_{0}-\tau_{0 c}[14]$ allows us to express the particle velocity $v_{p}$ as $v_{p}^{2} \sim v_{f}^{2}-$ 
$v_{f c}^{2}$, where $v_{f}$ is the near-bed flow velocity. Since the $v_{f}$ is provided by the localised eddy, the $v_{f}$ can be scaled as $v_{f} \sim U$. It results in

$$
v_{p} \sim\left(U^{2}-U_{c}^{2}\right)^{1 / 2} \sim U_{c}\left(\frac{\Theta}{\Theta_{c}}-1\right)^{1 / 2}
$$

Using Eq. (5), Eq. (7) produces

$$
v_{p} \sim(\Delta g d)^{1 / 2} \zeta^{(1+\sigma) / 4} G^{1 / 2}\left(D_{*}\right)\left(\frac{\Theta}{\Theta_{c}}-1\right)^{1 / 2}
$$

Substituting Eqs. (6) and (8) into the relationship $q_{b} \sim n d^{3} v_{p}$ yields

$$
\Phi_{b} \sim \frac{\left(\Theta-\Theta_{c}\right)^{3 / 2}}{\Theta_{c}^{1 / 2}} \zeta^{(1+\sigma) / 4} G^{1 / 2}\left(D_{*}\right) \sim T_{*}^{3 / 2} \zeta^{(1+\sigma) / 4} G^{3 / 2}\left(D_{*}\right)
$$

where $\Phi_{b}\left[=q_{b} /\left(\Delta g d^{3}\right)^{1 / 2}\right]$ is the bedload transport intensity and $T_{*}\left[=\left(\tau_{0}-\tau_{0 c}\right) / \tau_{0 c}=(\Theta-\right.$ $\left.\left.\Theta_{c}\right) / \Theta_{c}\right]$ is the transport stage parameter. The " $3 / 2$ " scaling law with $T_{*}$ completely corroborates the empirical formulas proposed earlier [16-19]. However, for $\sigma=-3,-5 / 3$ and -1 , the $\Phi_{b}$ follows the " $-1 / 2$ ", " $-1 / 6$ " and " 0 " scaling laws with the relative roughness, respectively.

\section{Scaling law of scour in a contracted stream}

(a)

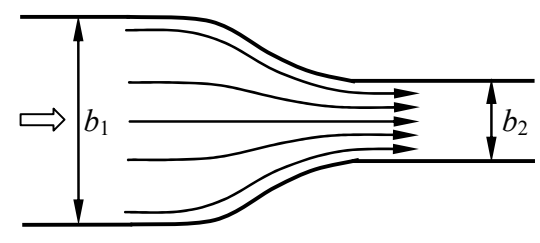

(b)

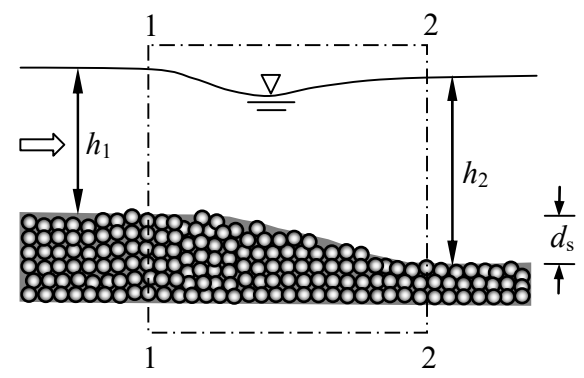

Fig. 3. Sketch of scour in a contracted stream: (a) plan view and (b) elevation view.

Scour in a contracted stream is one of the fascinating problems of sediment transport. The flow is accelerated convectively in the contracted portion because of the concentration of streamlines in the contraction. This yields a higher flow velocity that erodes the sediment bed in the contracted portion.

Figure 3 shows a schematic representation of scour in a channel contraction. The approach channel width and the approach flow depth are $b_{1}$ and $h_{1}$, respectively. On the other hand, the contracted channel width and the contracted flow depth are $b_{2}$ and $h_{2}$, respectively. When the approach flow enters into the contracted portion, the scour hole within the contraction is gradually developed and eventually, an equilibrium scour is 
reached when the flow velocity in the contracted channel reduces to the threshold velocity of sediment particles.

The continuity equation of fluid flow is expressed as

$$
U_{1} h_{1} b_{1}=\left.U_{2}\right|_{U_{2}=U_{c}} h_{2} b_{2}
$$

Neglecting the energy loss through the transition and the difference in velocity heads between the approach section and the contracted section produces $d_{s}=h_{2}-h_{1}$. Introducing the channel contraction ratio $r\left(=B_{2} / B_{1}\right)$, Eq. (10) produces

$$
\frac{D}{h_{1}}=\frac{d_{s}+h_{1}}{h_{1}}=\frac{U_{1}}{\left.U_{2}\right|_{U_{2}=U_{c}}} \frac{1}{r}
$$

The threshold velocity in the contracted channel can be obtained from Eq. (5) as

$$
\left.U_{2}\right|_{U_{2}=U_{c}} \sim(\Delta g d)^{1 / 2}\left(\frac{d}{h_{2}}\right)^{(1+\sigma) / 4} G^{1 / 2}\left(D_{*}\right)
$$

Substituting Eq. (12) into Eq. (11) yields

$$
\frac{D}{h_{1}} \sim F_{d 1}^{4 /(3-\sigma)} r^{-4 /(3-\sigma)} \zeta_{1}^{-(1+\sigma) /(3-\sigma)} G^{-2 /(3-\sigma)}\left(D_{*}\right)
$$

where $F_{d 1}\left[=U_{1} /(\Delta g d)^{1 / 2}\right]$ is the densimetric Froude number for the approach flow and $\zeta=$ $d / h_{1}$. For $\sigma=-3,-5 / 3$ and -1 , Eq. (13) produces $D / h_{1} \sim F_{d 1}^{2 / 3} r^{-2 / 3} \zeta_{1}^{1 / 3}, F_{d 1}^{6 / 7} r^{-6 / 7} \zeta_{1}^{1 / 7}$ and $F_{d 1} r^{-1} \zeta_{1}^{0}$, respectively. Note that $\sigma=-3$ indicates the limit of the hydraulically smooth flow, the experimental data can hardly be obtained for this case as most of the laboratory and field experimental data related to the context of scour problems belong to hydraulically transitional and/or rough flow regimes. Figure 4(a) shows the comparison of the proposed scaling law of $D / h_{1}$ for $\sigma=-5 / 3$ with the experimental data of several researchers [20-24]. These experimental data contain sand and gravel with the relative roughness belonging to $10^{-4}<\zeta_{1}<10^{-1}$, which justifies the validity of the spectral exponent $\sigma=-5 / 3$. The $\pm 20 \%$ error band is also shown for an understanding of the experimental data scatter from the best fitted straight line. On the other hand, Fig. 8(b) shows the comparison of the proposed scaling law of $D / h_{1}$ for $\sigma=-1$ with the experimental data of Dey and Raikar [24]. These experimental data contain large gravels with the relative roughness belonging to $10^{-1}<\zeta_{1}<$ 1 , which justifies the existence of $\sigma=-1$. The $\pm 20 \%$ error band is also shown. Figure 8 shows that the proposed scaling law of $D / h_{1}$, in general, has a satisfactory agreement with the experimental data. 

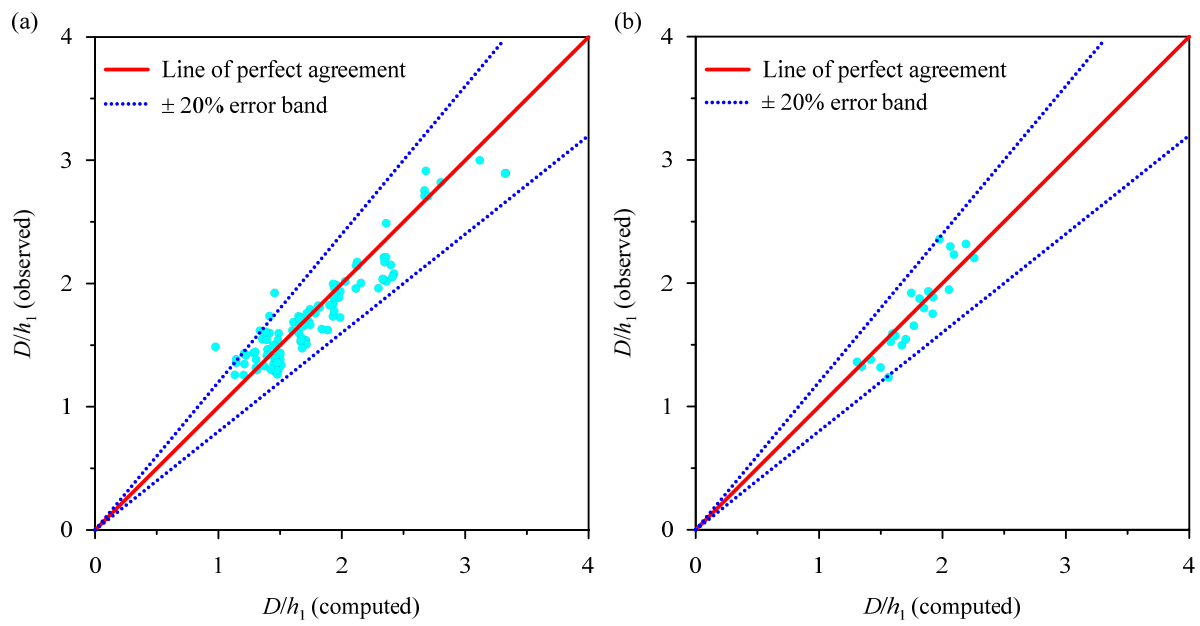

Fig. 4. Comparison of the scaling laws of $D / h_{1}$ for (a) $\sigma=-5 / 3$ with the experimental data [20-24] and (b) $\sigma=-1$ with the experimental data [24].

Importantly, the empirical formula of Straub [25] shows that $D / h_{1} \sim r^{-6 / 7}$, which is in agreement with the present study for $\sigma=-5 / 3$. Moreover, for $\sigma=-5 / 3$, the proposed scaling law of $D / h_{1}$ completely corresponds to the formulae of Gill [21] and Laursen [26].

\section{Conclusion}

The scaling laws of sediment transport under turbulent flow are sought from the perspective of the phenomenological theory of turbulence. This theory provides a close association between the scaling laws of sediment transport and the spectral laws of turbulence. This study derives the scaling laws of three classical problems of sediment transport: the threshold of sediment motion, the bedload transport rate and the scour in a contracted stream. It is revealed that the phenomenological theory provides an easy means to obtain the origin of these scaling laws. It also provides an insight into the sediment transport problems by explaining the interaction between the sediment particles and the localised turbulence.

\section{References}

1. A. N. Kolmogorov, Dokl. Akad. Nauk SSSR 30, 299-303 (1941)

2. U. Frisch, Turbulence: the legacy of A. N. Kolmogorov (Cambridge University Press, 1995)

3. G. Gioia, F. A. Bombardelli, Phys. Rev. Lett. 88, 014501 (2002)

4. R. H. Kraichnan, Phys. Fluids 10, 1417-1423 (1967)

5. V. Nikora, Phys. Rev. Lett. 83, 734-736 (1999)

6. S. Dey, Fluvial hydrodynamics: hydrodynamic and sediment transport phenomena (Springer, 2014)

7. L. L. Lischtvan, V. V. Lebediev, Gidrologia i Gidraulika v Mostovom Doroshnom, Straitielvie. Leningrad (Hydrology and Hydraulics in Bridge and Road Building), Gidrometeoizdat, Leningrad (1959) 
8. C. R. Neill, Proc. of the 12th congress of International Association for Hydraulic Research, Fort Collins, Colorado, USA, vol 3, pp. 46-54 (1967)

9. K. Ashida, M. Bayazit, Proc. of the 15th congress of International Association for Hydraulic Research, Istanbul, Turkey, vol 1, pp. 475-484 (1973)

10. M. L. Olivero, Application Rep. for Associate Professor, Univ. de Los Andes, Mérida, Venezuela, pp. 169 (1984)

11. J. Aguirre-Pe, R. Fuentes, Proc. of the 24th congress of International Association for Hydraulic Research, Madrid, Spain, pp. 149-158 (1991)

12. J. C. Bathurst, W. H. Graf, H. H. Cao, Mechanics of sediment transport (B. M. Summer and A. Müller, eds., Rotterdam, The Netherlands, pp. 207-213, 1983)

13. J. C. Bathurst, H. H. Cao, W. H. Graf, Ecole Polytechnique Fédérale de Lausanne (EPFL) Rep. CH-1015, Lausanne, Switzerland, 64 (1984)

14. R. A. Bagnold, Phil. Trans. R. Soc. Lond. A 249, 235-297 (1956)

15. F. Engelund, J. Fredsøe, Nord. Hydrol. 7, 293-306 (1976)

16. H. A. Einstein, Technical bulletin 1026, United States Department of Agriculture, Soil Conservation Service, Washington DC (1950)

17. E. Meyer-Peter, R. Müller, Proc. of the 2nd meeting of International Association for Hydraulic Research, Stockholm, Sweden, vol 3, pp. 39-64 (1948)

18. R. Fernandez Luque, R. van Beek, J. Hydraul. Res. 14, 127-144 (1976)

19. L. C. van Rijn, J. Hydraul. Eng. 110, 1494-1502 (1984)

20. S. Komura, J. Hydraul. Div. 92, 17-37 (1966)

21. M. A. Gill, J. Hydraul. Div. 107, 273-284 (1981)

22. M. G. Webby, RRU bulletin 73, National Roads Board, Bridge Design and Research Seminar, New Zealand, pp. 109-118 (1984)

23. S. Y. Lim, Proc. Inst. Civ. Engrs Wat. Marit. Energy 101, 93-98 (1993)

24. S. Dey, R. V. Raikar, J. Hydraul. Eng. 131, 1036-1049 (2005)

25. L. G. Straub, Trans. Am. Geophys. Union 15, 454-463 (1934)

26. E. M. Laursen, J. Hydraul. Div. 89, 93-118 (1963) 